\title{
An Emergency Medicine-focused Summary of the HFSA/SAEM/ISHLT Clinical Consensus Document on the Emergency Management of Patients With Ventricular Assist Devices
}

Chad E. Darling, $\mathrm{MD}^{1}$ (D), Jennifer L. Martindale, $\mathrm{MD}^{2}$, Brian C. Hiestand, MD ${ }^{3}$, Mark Bonnell, $\mathrm{MD}^{4}$, Monica Colvin, $\mathrm{MD}^{5}$, Ersilia M. DeFilippis, $\mathrm{MD}^{6}$, Tonya Elliott, RN, MSN 7 , Eman Hamad, $\mathrm{MD}^{8}$, Sean P. Pinney, MD ${ }^{9}$, Keyur B. Shah, $\mathrm{MD}^{10}$, Juliane Vierecke, $\mathrm{MD}^{11}$, and Michael M. Givertz, $\mathrm{MD}^{6}$

\section{ABSTRACT}

Mechanical circulatory support is increasingly used as a long-term treatment option for patients with end-stage heart failure. Patients with implanted ventricular assist devices are at high risk for a range of diverse medical urgencies and emergencies. Given the increasing prevalence of mechanical circulatory support devices, this expert clinical consensus document seeks to help inform emergency medicine and prehospital providers regarding the approach to acute medical and surgical conditions encountered in these complex patients.

$\mathrm{M}$ echanical circulatory support is a viable longterm treatment option for patients with endstage heart failure. As the range of indications for implantation of ventricular assist devices grows, so does the number of patients who live with durable support. These patients are at high risk for medical urgencies and emergencies (Table 1). This article is an emergency medicine-focused summary of a consensus document written collaboratively by the Heart Failure Society of America (HFSA), Society for Academic Emergency Medicine (SAEM), and International Society for Heart and Lung Transplantation (ISHLT). ${ }^{1}$ It aims to educate emergency medicine and prehospital providers managing patients with left ventricular assist devices (LVADs).

\section{OVERVIEW OF VENTRICULAR ASSIST DEVICES}

Mechanical circulatory support is a major advance in the treatment of patients with end-stage heart failure. ${ }^{2}$ Currently, there are three recognized indications for the use of LVADs: 1) bridge to transplantation (BTT), 2) destination therapy for patients ineligible for heart transplant, and 3) bridge to myocardial recovery. ${ }^{3}$

From the ${ }^{1}$ UMass Memorial Medical Center, UMass Medical School, Worcester, MA; ${ }^{2}$ lcahn School of Medicine at Mount Sinai, New York, NY; ${ }^{3}$ Wake Forest School of Medicine, Winston-Salem, NC; ${ }^{4}$ Mclaren Greater Lansing, Lansing, MI; ${ }^{5}$ University of Michigan Medical Center, Ann Arbor, Ml; ${ }^{6}$ Brigham and Women's Hospital, Harvard Medical School, Boston, MA; ${ }^{7}$ MedStar Washington Hospital Center, Washington, DC; ${ }^{8}$ Temple University Hospital, Philadelphia, PA; ${ }^{9}$ Mount Sinai Medical Center, New York, NY; ${ }^{10}$ VCU Pauley Heart Center, Richmond, VA; ${ }^{11}$ University of Cincinnati College of Medicine, Cincinnati, $\mathrm{OH}$.

Received November 14, 2019; revision received February 24, 2020; accepted March 10, 2020.

The authors have no relevant financial information or potential conflicts to disclose.

Supervising Editor: Anna Marie Chang, MD.

Address for correspondence and reprints: Michael M. Givertz, MD; e-mail: mgivertz@bwh.harvard.edu.

ACADEMIC EMERGENCY MEDICINE 2020;27:618-629. 
Table 1

Common Emergencies in Patients With VADs

\begin{tabular}{lll}
\hline VAD specific emergencies & VAD related emergencies & VAD unrelated emergencies \\
\hline Heart failure & Arrhythmias & Abdominal pain \\
Left heart failure & Atrial fibrillation & Blunt and penetrating trauma \\
Right heart failure & Ventricular tachycardia & Burns \\
Mechanical failure & Ventricular fibrillation & Hypovolemia \\
Driveline & Bleeding & Infection \\
Pump stoppage & Epistaxis & Sepsis \\
Pump thrombosis & Gastrointestinal & \\
& Cardiac arrest & \\
& Cardiac tamponade & \\
& Infection & \\
& Driveline & \\
& Pump pocket & \\
& Stroke & Hemorrhagic \\
\hline
\end{tabular}

VAD $=$ ventricular assist device.

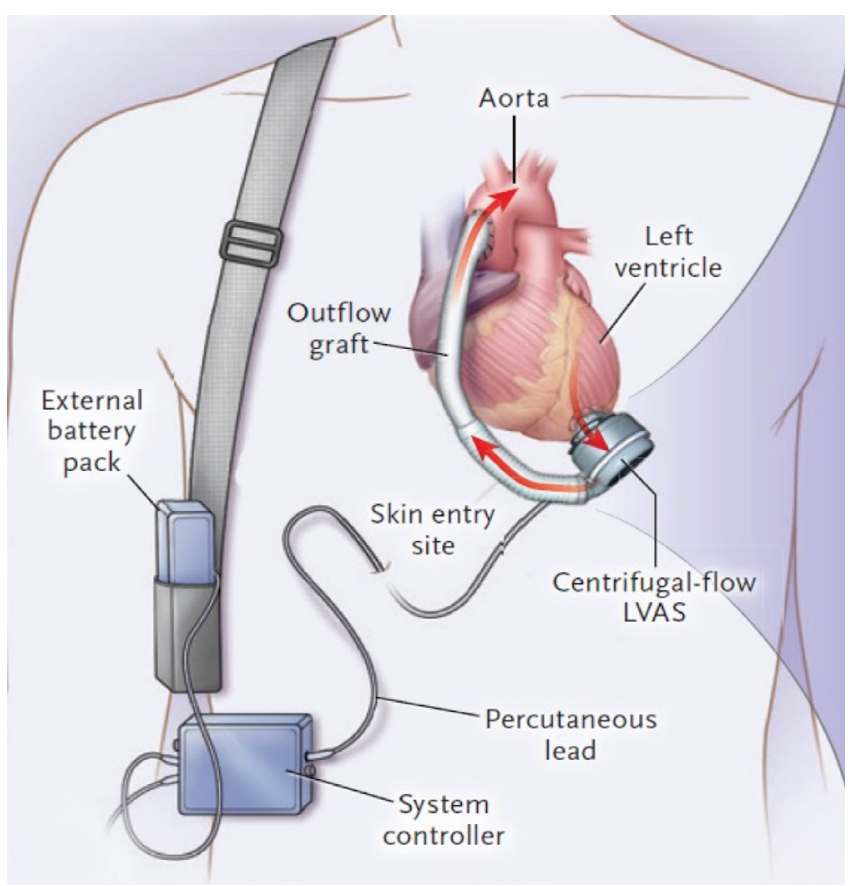

Figure 1. Components of a typical LVAD. A CF LVAD consists of a pump connected to the cardiac apex and ascending aorta via an inflow cannula and outflow graft, respectively; a percutaneous driveline that exits the skin on the right; and a system controller that is typically worn on a belt. Power to the controller and pump is provided by external batteries or a power-based unit. Adapted from Mehra et al. ${ }^{7}$ with permission. CF $=$ continuous flow; LVAD $=$ left ventricular assist device.

\section{Volumes and Survival}

According to the eighth annual Interagency Registry for Mechanically Assisted Circulatory Support (INTERMACS) report, there were a total of 22,866 VAD implants from June 2006 to December 2016. Of these, 18,987 were primary implants for left ventricular support. ${ }^{4}$

Left ventricular assist devices offer superior survival when compared to optimal medical management in patients who are ineligible for cardiac transplantation. For continuous-flow (CF) devices implanted since 2008 , the overall 1 -year survival is $81 \%$ and 2 -year survival is $70 \%{ }^{5}$

\section{LVAD "Anatomy"}

Contemporary LVADs consist of an "inflow" cannula that drains the left ventricle and an "outflow" graft to a central artery-usually the ascending or descending aorta. Other internal components consist of the pump and part $(20-30 \mathrm{~cm})$ of the driveline, containing wires that power and control the pump. The driveline typically exits through the upper abdominal wall. External components are a controller and an external power source (Figure 1). Most current LVADs provide CF through a rotary pump, resulting in blood flow with reduced or no pulsatility. ${ }^{6}$ However, the HeartMate 3 (Abbott) is a fully magnetically levitated pump that provides an artificial pulse (once every 2 seconds, the pump modifies its speed) that was approved for BTT in $2017 .^{7,8}$

\section{CLINICAL ASPECTS SPECIFIC TO LVAD PATIENTS}

\section{Assessing Equipment}

While evaluating a patient with an LVAD, one should determine the make and model of the pump. All LVADs have an external driveline connected to a control device and power source. Locate the driveline, which allows the pump to receive information and power to run the LVAD system. This cable should be attached at one end to the implanted pump and connected to the controller. 
The dressing on the abdominal wall where the driveline exits the skin should be dry and intact. Patients will wear either an abdominal binder or an anchor device to secure the driveline. Look under binders and dressings to inspect the entirety of the line. The anchor or binder should be kept in place during transport and treatment.

The size and configuration of controllers differ by manufacturer. The controller communicates with the pump and displays pump parameters (e.g., speed, flow, power) and alarms for both advisory and hazardous conditions. The controller accommodates two power sources - either dual batteries or one battery plus an AC/DC adapter. Dual batteries can provide anywhere from 8 to 12 hours of support while patients are active. The percentage of charge remaining in each battery can be determined by pushing the button on the top of the battery.

All patients should have an extra controller and batteries as backup equipment. Exchange the primary controller (attached to the patient) for the backup controller only when indicated by controller alarms, such as "controller fault, change controller" and with the guidance of a provider trained to perform this exchange. Prehospital personnel are advised to transport a patient's backup LVAD equipment.

\section{LVAD Parameters}

Pump function and flow, displayed on the controller, are the "vital signs" of the LVAD. A typical display shows blood flow (liters/min), pump rotary speed (rotations/min), and power consumption (Watts). LVAD flow is calculated based on rotary speed and power consumption and approximates cardiac output.

\section{Device Alarms}

The controller has visual and auditory alarms that indicate problems with the pump, controller, connections, or power supply. Address alarms by first looking at the controller and reading the condition. Contacting the implant center or the manufacturer's clinical specialist and/or accessing online support are critical for managing alarms.

\section{Antithrombotic Therapy}

Antithrombotic therapy is necessary for all patients with VADs. Typically, patients are maintained on warfarin with an international normalized ratio (INR) target of 2.0 to 3.0 and 81 to $325 \mathrm{mg}$ of aspirin daily. Some VAD programs use dipyridamole or clopidogrel as additional antiplatelet therapy (for example, in patients with a history of threatened pump thrombosis or transient ischemic attack), but there are no supportive data. Reversal of warfarin with vitamin $\mathrm{K}$, fresh-frozen plasma, or prothrombin concentrate complex may be considered when treating life-threatening bleeding events or preparing for emergent surgery.

\section{Patient Assessment: Vital Signs}

The ability to obtain vital signs in LVAD recipients depends on the extent that remaining native ventricular function can generate pulsatile blood flow. For most patients, the absence of a pulse is a normal finding and differences in systolic and diastolic blood pressure are undetectable by automatic and manual sphygmomanometers. ${ }^{9}$ A vascular Doppler can be used to assess blood pressure - it is commonly assumed that the first sound heard approximates the mean arterial pressure (MAP), yet studies show this may be closer to the systolic pressure. ${ }^{10}$ Regardless, we suggest using the Doppler opening pressure as a surrogate for MAP, with current ISHLT guidelines recommending a mean blood pressure goal of $\leq 80 \mathrm{~mm} \mathrm{Hg} .{ }^{11}$ Oxygen saturation is another vital sign that might be inaccurate, as pulse oximetry depends on pulsatile flow. ${ }^{12}$ Absent a typical pulse oximetry waveform, the result is likely inaccurate. Clinicians must rely on direct assessments of mental status, perfusion, and general appearance to gauge the clinical condition of LVAD recipients.

\section{Clinical Assessment}

Important elements of patient history include preceding symptoms (e.g., shortness of breath, chest pain, headache, blood in the urine or stool), the model of their LVAD, and alarm history. Patients may be able to identify their own equipment malfunctions or driveline concerns.

Assessment of airway patency, work of breathing, and adequacy of perfusion are paramount. Clinicians should be able to auscultate a mechanical hum and feel vibrations generated by the LVAD. Beyond initial assessment, clinicians should specifically look for physical signs of heart failure, decreased peripheral perfusion, infection, and blood loss.

\section{EMERGENCY MANAGEMENT SYSTEM CHALLENGES}

\section{Field Assessment and Transport}

Sending patients who are pulseless and device-dependent into the community requires coordination of the 
Table 2

Echocardiographic Findings in LVAD Emergencies

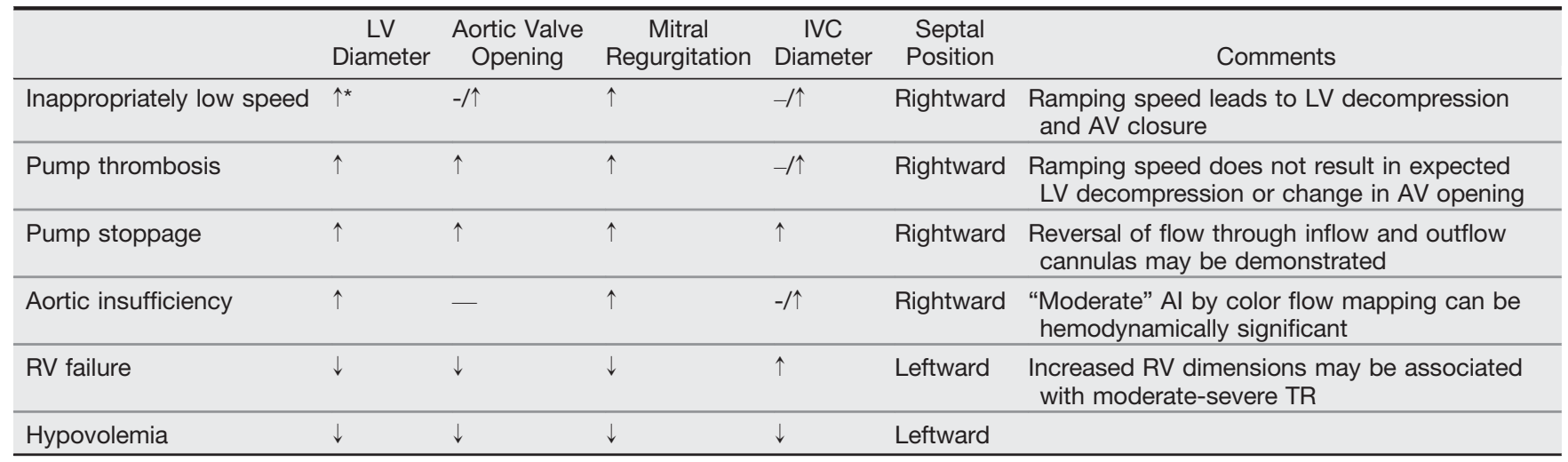

$\mathrm{Al}=$ aortic insufficiency; $\mathrm{AV}=$ aortic valve; IVC = inferior vena cava; LV = left ventricular; LVAD = left ventricular assist device; RV = right ventricular; $\mathrm{TR}=$ tricuspid regurgitation.

*Increase or decrease relative to baseline study obtained when patient clinically stable.

community and prehospital providers with the implanting center. ${ }^{13}$ As part of the certification process, LVAD centers are required to provide first-responder education and coordination plans. ${ }^{11}$ Current versions of the field guides for LVADs can be accessed online at https://www.mylvad.com/medicalprofessionals/resource-library/ems-field-guides.

When LVAD patients are transported to community hospitals, the on-site team should contact the LVAD center as soon as clinically possible. A collaborative course of action can then be implemented that best serves the patient by determining whether the specific problem can be dealt with in a community hospital or requires transfer.

\section{MEDICAL EMERGENCIES IN PATIENTS WITH LVADS}

\section{Cardiac Arrest}

In patients with CF LVADs, cardiac arrest may be difficult to ascertain clinically or to differentiate from other conditions resulting in syncope or impaired consciousness. As a result, cardiac arrest resuscitation is often delayed in patients with LVADs compared to other medical patients. ${ }^{14}$ In the unconscious patient, the absence of mechanical hum on precordial auscultation, inability to obtain a Doppler signal on manual blood pressure measurement, and cardiac standstill on echocardiography are diagnostic of cardiac arrest. In the prehospital setting, a patient who is unresponsive and apneic, and in whom a mechanical hum cannot be auscultated, should be assumed to be in cardiac arrest and receive advanced cardiac life support (ACLS). Assessment of cardiac rhythm with a portable monitor or electrocardiogram (ECG) is indicated in all patients, although the LVAD may cause significant artifact due to electrical interference.

There are varying opinions regarding both efficacy and safety of chest compressions in patients with LVADs, with the debate informed by case series ${ }^{15}$ and retrospective cohort studies. ${ }^{14,16}$ Primary safety concerns include damage to or dislodgement of the cannula or displacement of the pump, resulting in catastrophic failure and potential intrathoracic exsanguination. Neurologically intact survival without device damage is possible following cardiac arrest in LVAD recipients, ${ }^{15}$ and the American Heart Association recommends bystander CPR for LVAD recipients. ${ }^{17}$ However, based on the lack of evidence of efficacy and equipment concerns, we do not recommend routine use of mechanical CPR devices.

\section{Unstable Arrhythmias}

Arrhythmias, both atrial and ventricular, are common and seldom life-threatening in patients with an LVAD. Ventricular tachycardia (VT) is common and results from underlying cardiomyopathy, right ventricular (RV) failure, or mechanical decompression (e.g., suction) of the ventricle by the inflow cannula. Because of the degree of physiologic support afforded by the LVAD, patients may complain only of fatigue, nausea, or light-headedness caused by arrhythmias that would be otherwise fatal. ${ }^{18}$ Therefore, ECGs should be obtained, even when the patient's chief complaint is not overtly cardiac in nature.

Clinicians treating VT or ventricular fibrillation (VF) in the LVAD recipient are typically afforded more time to pursue an underlying cause and consider different treatment options. If point-of-care 
echocardiography is available, examination of the inflow cannula position within the ventricle may demonstrate the presence of an overly decompressed LV with collapse and marked septal shift, which may trigger arrhythmias due to myocardial irritation. This collapse may respond to decreasing LVAD speed, allowing for increased ventricular filling and migration of the septum away from the inflow cannula. An intravenous fluid bolus can augment preload and limit systolic ventricular collapse against the cannula during systole as a temporizing measure in hypovolemic patients regardless of the degree of RV dysfunction. Table 2 summarizes abnormal echocardiographic findings in LVAD emergencies.

Arrhythmias resulting in severe hemodynamic instability should be treated according to standard ACLS protocols. While many patients with LVADs will have an implantable cardioverter-defibrillator (ICD), for those without ICDs, standard cardioversion and defibrillation are not contraindicated and may be performed without disconnection from the device. If possible, the pads should not be placed directly over the device itself-anterior/posterior placement is preferred. Antiarrhythmic agents such as amiodarone should be considered in the absence of a mechanical cause of the arrhythmia if the patient is hemodynamically stable. However, in refractory or hemodynamically significant ventricular arrhythmias, cardioversion/defibrillation will often be required. ${ }^{18}$

\section{Myocardial Infarction}

Acute myocardial infarction (MI) can occur in patients with LVADs due to plaque rupture from underlying coronary artery disease or coronary embolism from ventricular or aortic root thrombus. Aortic root thrombus tends to occur early postimplant often in the setting of a subtherapeutic INR $^{19}$ and can be visualized by transesophageal echocardiography (TEE) or computed tomography (CT) angiography. ${ }^{20}$ Depending on the cause of acute MI, management may include percutaneous coronary intervention, intensification of anticoagulation, or aortic root thrombectomy. ${ }^{21}$ Since patients are typically fully supported by the LVAD, chest pain may be the only presenting symptom with heart failure being less common.

\section{Unexplained Hypotension}

Left ventricular assist systems do not have a direct way to measure the amount of blood in the left ventricle, making patients vulnerable to low flow complications and hypotension (defined as a MAP or Doppler

Table 3

Differential Diagnosis and Management of Unexplained Hypotension

\begin{tabular}{|c|c|c|}
\hline Cause & Signs & Intervention \\
\hline $\begin{array}{l}\text { Bleeding (gastrointestinal, } \\
\text { nasal and cerebral } \\
\text { hemorrhage) }\end{array}$ & $\begin{array}{l}\text { Low hemoglobin, hematocrit, and } \\
\text { platelet count } \pm \text { elevated INR }+ \text { stool } \\
\text { guaiac } \\
\text { Low flow }\end{array}$ & $\begin{array}{l}\text { Bolus IV fluids, transfusion } \\
\text { Hold or reverse anticoagulation } \\
\text { Identify and treat bleeding source }\end{array}$ \\
\hline $\begin{array}{l}\text { Dehydration (infection, } \\
\text { vomiting, diuretics, poor } \\
\text { oral intake) }\end{array}$ & $\begin{array}{l}\text { Low JVP } \\
\text { Low flow/low PI } \pm \text { suction }\end{array}$ & $\begin{array}{l}\text { IV fluids, hold diuretics } \pm \text { decrease VAD speed } \\
\text { temporarily to avoid suction } \\
\text { Identify etiology and treat }\end{array}$ \\
\hline Inadequate $L V A D$ speed & $\begin{array}{l}\text { High JVP } \\
\text { Low flow }\end{array}$ & $\begin{array}{l}\text { Echo and RHC } \\
\text { Inadequate unloading by LVAD: high PCWP, low output } \\
\text { Adjust pump speed }\end{array}$ \\
\hline Arrhythmia & $\begin{array}{l}\text { Obtain rhythm strip immediately } \\
\text { Sudden cardiac arrest may be difficult } \\
\text { to define as VAD patients can be } \\
\text { awake while in VF } \\
\text { Low flow } \pm \text { suction }\end{array}$ & Use ACLS guidelines to treat arrhythmia \\
\hline $\begin{array}{l}\text { Sepsis (driveline exit site, } \\
\text { indwelling catheters or } \\
\text { home IVs) }\end{array}$ & $\begin{array}{l}\text { Elevated WBC, fever } \\
\text { High flow due to low SVR }\end{array}$ & $\begin{array}{l}\text { Hold vasodilators } \\
\text { Add pressor support } \\
\text { Identify source and treat }\end{array}$ \\
\hline
\end{tabular}

ACLS = advanced cardiac life support; CTA = computed tomographic angiography; INR = international normalized ratio; IV = intravenous; $\mathrm{JVP}=$ jugular venous pressure; LDH = lactate dehydrogenase; LVAD = left ventricular assist device; MAP = mean arterial pressure; $\mathrm{PCWP}=$ pulmonary capillary wedge pressure; $\mathrm{PE}=$ pulmonary embolism; $\mathrm{PH}=$ pulmonary hypertension; $\mathrm{RHC}=$ right heart catheterization; SVR = systemic vascular resistance; VF = ventricular fibrillation; WBC = white blood cell count. 


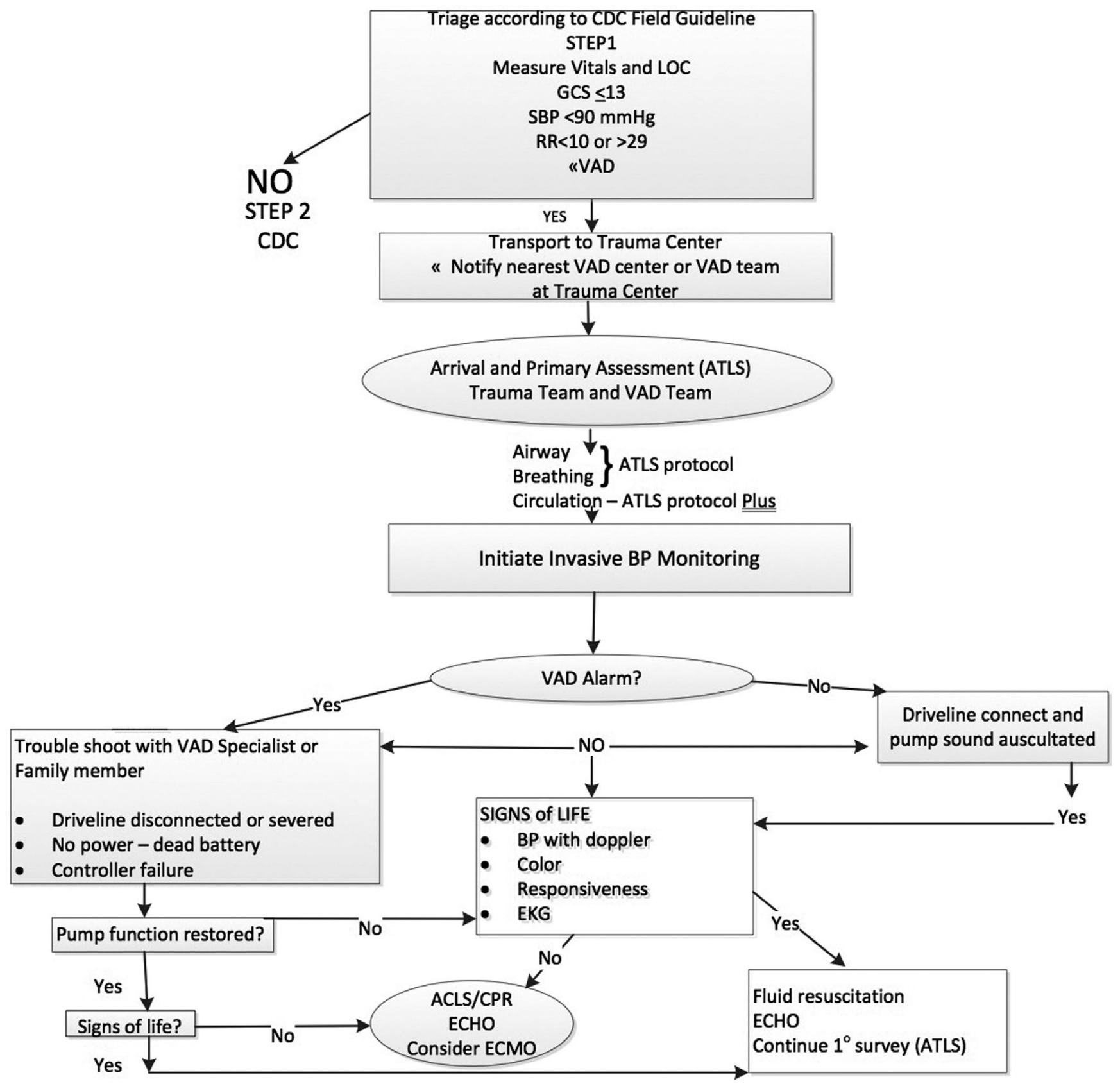

Figure 2. Proposed algorithm for management of trauma in a patient with an LVAD. ATLS = Advanced Trauma Life Support; BP = blood pressure; CDC, Centers for Disease Control and Prevention; CPR = cardiopulmonary resuscitation; ECHO = echocardiogram; ECMO = extracorporeal membrane oxygenation; EKG = electrocardiogram; GCS = Glasgow Coma Scale; LOC = level of consciousness; LVAD = left ventricular assist device; $\mathrm{RR}$ = respiratory rate; SPB = systolic blood pressure; VAD = ventricular assist device.

opening pressure $<60 \mathrm{~mm} \mathrm{Hg}$ for a CF device). Factors resulting in preload reduction (e.g., hypovolemia, RV failure) lead to suboptimal LV filling, which in turn can cause suboptimal flow or suction in the inflow cannula. The pump will continue to spin with a minimal ability to reduce speed to compensate for decreased volume, and instability can ensue. Multiple conditions, including sepsis, arrhythmia, pulmonary embolism, and hypovolemia, can reduce LVAD preload leading to a low-flow alarm (Table 3). In addition, pump thrombosis or cannula obstruction can also impair device flow. ${ }^{11}$

Low flow in the device accompanied by increasing central venous pressure can suggest RV failure. ${ }^{22}$ In the subacute and chronic settings, RV dysfunction can be due to ventricular arrhythmias, volume overload, pulmonary embolism (if INR is subtherapeutic), persistent pulmonary hypertension, or tricuspid regurgitation. $^{23}$ Excessive pump speed and flow can also overwhelm an already compromised right ventricle at 
any time following LVAD implant. In addition, use of anesthetic agents with negative inotropic properties (e.g., propofol) should be avoided in patients with marginal or reduced RV function. RV failure can lead to hemodynamic deterioration, ICD shocks, and even cardiac arrest with VT/VF caused by impaired filling and inadequate LVAD flow. In all low-flow cases, an echocardiogram should be urgently obtained to assess RV and LV dimensions and filling and rule out tamponade. $^{11}$ If the pump stops (see below) and the patient is in cardiogenic shock, vasopressors along with inotropes may be needed to support diminished heart function.

\section{Blunt and Penetrating Trauma}

Patients with LVADs should receive the same initial trauma evaluation as any patient $\mathrm{t}^{24,25}$ in concert with VAD system troubleshooting and cardiac surgery consultation as needed. While current ATLS protocols do not account for mechanical circulatory support, a proposed accessory algorithm is presented (Figure 2). There is ample evidence that patients who are anticoagulated are at higher risk following trauma, but there is no consensus regarding the management of anticoagulation in trauma, let alone in patients with LVADs. Invasive blood pressure monitoring should be considered early in the clinical course. If available, the LVAD team should be consulted to ensure proper device function. A standard chest x-ray helps to verify pump position and basic integrity of the driveline, but a targeted $\mathrm{x}$-ray of the driveline should be performed to ensure there has been no break in the wires. If so, the manufacturer representative should be notified immediately as most extracorporeal wire fractures can be safely repaired at the bedside. ${ }^{26}$

Point of care ultrasound can evaluate for pericardial effusion in the trauma patient, but formal echocardiography is recommended to identify proper pump placement, disturbances in the blood flow pathway, and abnormal RV function. In addition to standard laboratory tests, lactate dehydrogenase (LDH) or plasma-free hemoglobin levels should be obtained to assess for hemolysis, which may be clues to more subtle disturbances in the blood flow pathway. Decisions regarding imaging and surgical management should be based on both hemodynamic stability and functional status of the LVAD. If the patient is hemodynamically stable, he or she can be closely monitored in an LVAD-capable intensive care unit or step-down unit. If the patient is hemodynamically unstable, one should proceed to surgery and the pump should be assessed for salvageability.

\section{Abdominal Pain}

A patient with an LVAD presenting with abdominal pain presents unique challenges. Patients may have tenderness around the driveline or the pump pocket that may mask or mimic other intraabdominal processes. The presentation of a driveline or pump pocket infection (discussed below) may be mistaken for other diagnoses and must be considered in the differential. Radiologic examination should be guided by clinical judgment. CT maintains its broad utility for abdominal pathology, but artifact from the pump obscures some windows.

\section{Bleeding Complications}

Nonsurgical bleeding is a common cause of morbidity in patients supported with LVADs. The most common sources of bleeding include gastrointestinal (GI) and epistaxis, although intracranial and intrathoracic bleeding may also occur. ${ }^{27-29}$ Platelet dysfunction, lysis of the von Willebrand polymer, and RV dysfunction with hepatic congestion all contribute to bleeding complications during VAD support. ${ }^{30-32}$ The event rate of bleeding in these patients far exceeds those observed in patients anticoagulated for other reasons. ${ }^{28}$

Gastrointestinal bleeding occurs in approximately $27 \%$ of patients with CF LVADs. ${ }^{33,34}$ The most common etiology in these patients is arteriovenous malformations in either the stomach or the duodenum. The diagnostic yield of typical endoscopic procedures may be lower since many patients have a small bowel source of bleeding. ${ }^{35}$ If bleeding is not identified, push enteroscopy or other methods of evaluating the small bowel are recommended. ${ }^{33,35}$ A suggested algorithm for upper/lower GI bleeding in VAD patients has recently been published. ${ }^{35}$

Management of symptomatic GI bleeding is patients with VADs is challenging. Withholding or reversing anticoagulation drugs should be first discussed with the patient's LVAD team. Transfusion may increase pulmonary artery pressures and worsen RV function. In BTT patients, transfusion of leukoreduced blood is preferable to reduce the risk of allosensitization. The added benefit of octreotide and thalidomide in patients with angiodysplastic lesions has not been demonstrated, although some programs have incorporated these agents into management. ${ }^{36-40}$

Epistaxis is the second most common bleeding complication in VAD patients. ${ }^{28}$ Management is the same 
as with any other anticoagulated patient, including topical vasoconstriction, cautery, nasal packing, and embolization as needed. Early involvement of otolaryngology is advisable.

\section{Stroke}

Cerebrovascular complications remain one of the more common adverse events experienced by patients with LVADs. ${ }^{41,42}$ The incidence of stroke is approximately $10 \%$ per year ${ }^{4,43}$ with the risk for stroke being highest in the early postoperative period as well as 9 to 12 months after implantation. 4,44

If an acute neurologic deficit develops in a patient with an LVAD, emergent CT of the head with angiography of the head and neck and neurology consultation should be obtained. ${ }^{11}$ LVAD parameters should be reviewed for any signs of device malfunction or thrombosis. Hospitals without VAD programs should urgently discuss the clinical situation with the patient's LVAD specialist(s) to determine if urgent transfer is warranted, and in hemorrhagic strokes, to discuss discontinuation or reversal of anticoagulation. Reversal targets an INR $<1.5$, and prothrombin complex concentrate may be selected over fresh-frozen plasma for more rapid effect and to avoid excess volume. $^{45}$

In patients with ischemic stroke, selective use of systemic or intraarterial thrombolytic agents or an interventional neuroradiologic procedure (e.g., endovascular thrombectomy) may be considered, but in the absence of prospective data, neither is routinely recommended. Case reports of patients with thromboembolic stroke complicated by LVAD thrombosis have demonstrated safe and successful use of systemic thrombolysis. ${ }^{46}$

\section{Infection/Sepsis}

A 2011 ISHLT working group standardized the definitions of LVAD infections and classified them into LVAD-specific, LVAD-related, and non-LVAD-related. ${ }^{47}$ Non-LVAD-related infections are those not affected by the presence of the LVAD, but happen to occur in a patient with an LVAD such as urinary tract infection or pneumonia. These infections are treated in the usual fashion. LVAD-related infections refer to infections not involving the LVAD itself, but that can have different characteristics or implications and management when present in an LVAD patient. These include infective endocarditis, bacteremia and mediastinitis. ${ }^{48}$ LVAD-specific infections involve the driveline, pocket, pump, and/or cannula.
Approximately $14 \%$ to $35 \%$ of LVAD patients develop percutaneous driveline infections. ${ }^{6,49,50}$ The probability of developing an infection increases approximately $4 \%$ for each additional month of LVAD support. ${ }^{51}$ Infection of the percutaneous driveline can range from simple cellulitis to abscess formation and deep soft tissue infection. Pocket infections refer to infection of the space housing the pump. CT scan and/or ultrasound should be used to assess for fluid collections around the device. Needle aspiration can be performed, but would best be performed at the patient's primary LVAD center. The most common pathogens leading to device-related infection include Staphylococcus, Enterococcus, and Pseudomonas species. Fungal infections are rare, ${ }^{52}$ but may be caused by Candida species, with a few case reports of Aspergillus and other molds.

Guidelines for the management of LVAD-associated infections have been proposed and are based on the type and extent of the infection. ${ }^{52}$ Patients with documented device-related infection should be treated with targeted antibiotic therapy for 4 to 6 weeks and surgical intervention/debridement as needed. Central lines and ICD generators and leads should be removed according to current guidelines. ${ }^{53,54}$ LVAD removal or exchange is associated with significant morbidity in addition to the difficulty of removing all infected parts and having a device-free period to allow the antibiotics to take effect before reimplant.

\section{LVAD-SPECIFIC EMERGENCIES}

\section{Pump Thrombosis}

Pump thrombosis can occur for a number of reasons including inadequate anticoagulation, poorly controlled blood pressure, and inappropriately low pump flow. ${ }^{55}$ Lasting increases in pump power by greater than $50 \%$ from baseline may indicate thrombosis. Fibrin deposition can create drag, necessitating power increases to maintain pump speed. Transient power spikes or sustained increases in pump power accompanied by inaccurate, elevated flow estimates may be observed. Internal pump thrombosis may produce hemolysis and darkened or bloody urine. Hemolysis may be detected by elevations in serum LDH $(>600 \mathrm{mg} / \mathrm{dL}$ or 2.5 times baseline), elevated free hemoglobin (>40 mg/dL), or reduced haptoglobin levels. ${ }^{56}$ Heart failure symptoms are often present, although nonocclusive thrombi can result in significant hemolysis without causing hemodynamic instability. 
Pump thrombosis is a catastrophic LVAD emergency. Patients should be transported as soon as possible to the nearest LVAD center where pump exchange or lysis can be performed. Guidelines for the detection and management of suspected thrombosis have been published. ${ }^{57}$ Initial management begins by providing adequate levels of systemic anticoagulation, usually with unfractionated heparin or a direct thrombin inhibitor. Published reports of successful treatment with heparin or bivalirudin have led some to consider these interventions before considering surgery. ${ }^{58}$ Tissue plasminogen activator (TPA) use has been reported with mixed results, ${ }^{59,60}$ and we recommend against routine use. Pathologic studies have demonstrated that the clot is highly organized and unlikely to respond to thrombin breakdown, and therefore TPA should only be considered after contacting the implanting center. Although medical treatment with anticoagulant agents or fibrinolytic therapy can lead to clot resolution, the rate of recurrence is high. ${ }^{61}$

\section{Pump Stoppage or Failure and Driveline Trauma}

A little more than $3.5 \%$ of all deaths on LVAD support can be attributed to device malfunction. ${ }^{62}$ Failures can occur in the internal or external portion of the driveline, patient cable, or pump controller or with external power.

Pump stoppage occurs when there is a complete loss of power to the pump. This can arise due to depletion of battery power, disconnection of both power leads, or disconnection of the percutaneous lead from the controller. A constant, high-pitched alarm sounds and a "red heart" accompanies it on the pocket controller. This is an unstable situation that leads to severe regurgitation of blood from the aorta into the LV, because the pump does not contain valves to prevent retrograde flow. Patients will present with symptoms of acute heart failure. The pump will remain silent upon chest auscultation. Treatment requires restoring power to the pump even though doing so in patients with subtherapeutic anticoagulation risks thromboembolism and stroke.

Driveline trauma may result in pump stoppages. Repetitive flexing and bending of the percutaneous lead may result in short circuits that may temporarily or permanently stop the pump from rotating, accompanied by a constant alarm. ${ }^{63}$ Temporary or more durable repairs can sometimes be performed by an industry engineer specific to each device by soldering together disrupted wires depending on the location of the fracture (internal vs. external). ${ }^{26}$ Complete transection of the driveline will require urgent surgical pump replacement.

\section{Heart Failure With an LVAD}

Patients implanted with LVADs may present for emergency care of acute heart failure. Typically they present with subacute progression of symptoms or, less frequently, with sudden decompensation requiring emergent intervention. Several potential factors can reduce LVAD flow leading to congestion and a low cardiac output state. $^{23}$

Inadequate LV Decompression. Inadequate decompression of the LV can lead to heart failure in LVAD patients. Causative factors include: 1) suboptimal pump speed setting, 2) increased afterload, 3) obstruction to blood flow, 4) thrombosis of the pump impeller, or 5) aortic insufficiency. When the LVAD fails to adequately decrease LV volumes, imaging may reveal LV dilation, functional mitral valve regurgitation, and frequent aortic valve opening. Patients often experience clinical symptoms of fatigue and dyspnea and may have signs of congestion on exam. Patients with subacute symptoms presenting to non-LVAD centers may require transfer for speed adjustments, diuresis, and antihypertensive therapies.

Increased afterload is most frequently caused by poorly controlled blood pressure, which results in reduced pump output. The monitor will display decreased power consumption and low flow estimates. Extreme blood pressure increases can obliterate flow through the device, increasing the risk of cerebrovascular accidents and pump thrombosis.

Kinks or obstruction to inflow (sudden or gradual) or outflow cannula (gradual) can lead to heart failure with low power consumption and estimated flow displayed on the LVAD controller or monitor. Inflow cannula obstruction reduces preload to the LVAD and may occur gradually due to pannus formation or misalignment of the cannula due to LV remodeling or orientation of the pump pocket (i.e., changing abdominal girth). Abrupt cannula obstruction may present as a medical emergency with sudden heart failure, syncope, or shock. ${ }^{64,65}$

\section{SUMMARY AND FUTURE DIRECTIONS}

A growing number of adults with end-stage heart failure are living in the community with mechanical 
circulatory support. Like all community dwellers, patients with left ventricular assist devices may have urgent or emergent medical needs requiring hospitallevel care. This consensus document by the Heart Failure Society of America, Society for Academic Emergency Medicine, and International Society for Heart and Lung Transplantation aims to provide emergency and prehospital providers with information needed to understand the basics of continuous-flow pumps and how to handle patients and their equipment in emergency situations. More detailed information on specific devices is available online at manufacturers' websites or by contacting their clinical specialists. A list of left ventricular assist device implanting centers in the United States and Canada can also be found online at www.uab.edu/medicine/intermacs/enroll/currently-en rolled. Future studies will focus on management of device settings, fluid resuscitation, and anticoagulation around noncardiac surgery and trauma. The extrapolation of these emergency management guidelines to a small, but emerging population of pediatric patients living at home with left ventricular assist devices will also need to be explored.

\section{REFERENCES}

1. Givertz MM, DeFilippis EM, Colvin M, et al. HFSA/ SAEM/ISHLT clinical expert consensus document on the emergency management of patients with ventricular assist devices. J Card Fail 2019;25:494-515.

2. Stewart GC, Givertz MM. Mechanical circulatory support for advanced heart failure: patients and technology in evolution. Circulation 2012;125:1304-15.

3. Miller LW, Guglin M. Patient selection for ventricular assist devices: a moving target. J Am Coll Cardiol 2013;61:1209-21.

4. Kirklin JK, Pagani FD, Kormos RL, et al. Eighth annual INTERMACS report: special focus on framing the impact of adverse events. J Heart Lung Transplant 2017;36:10806.

5. Smith LA, Yarboro LT, Kennedy JL. Left ventricular assist device implantation strategies and outcomes. J Thorac Dis 2015;7:2088-96.

6. Slaughter MS, Rogers JG, Milano CA, et al. Advanced heart failure treated with continuous-flow left ventricular assist device. N Engl J Med 2009;361:2241-51.

7. Mehra MR, Naka Y, Uriel N, et al. A fully magnetically levitated circulatory pump for advanced heart failure. $\mathrm{N}$ Engl J Med 2017;376:440-50.

8. Uriel N, Colombo PC, Cleveland JC, et al. Hemocompatibility-related outcomes in the MOMENTUM 3 trial at 6 months: a randomized controlled study of a fully magnetically levitated pump in advanced heart failure. Circulation 2017;135:2003-12.

9. Myers TJ, Bolmers M, Gregoric ID, Kar B, Frazier OH. Assessment of arterial blood pressure during support with an axial flow left ventricular assist device. J Heart Lung Transplant 2009;28:423-7.

10. Lanier GM, Orlanes K, Hayashi Y, et al. Validity and reliability of a novel slow cuff-deflation system for noninvasive blood pressure monitoring in patients with continuousflow left ventricular assist device. Circ Heart Fail 2013;6:1005-12.

11. Feldman D, Pamboukian SV, Teuteberg JJ, et al. The 2013 International Society for Heart and Lung Transplantation Guidelines for mechanical circulatory support: executive summary. J Heart Lung Transplant 2013;32:157-87.

12. Oleyar M, Stone M, Neustein SM. Perioperative management of a patient with a nonpulsatile left ventricular-assist device presenting for noncardiac surgery. J Cardiothorac Vasc Anesth 2010;24:820-3.

13. Cook JL, Colvin M, Francis GS, et al. Recommendations for the use of mechanical circulatory support: ambulatory and community patient care: a scientific statement from the American Heart Association. Circulation 2017;135: e1145-58.

14. Garg S, Ayers CR, Fitzsimmons C, et al. In-hospital cardiopulmonary arrests in patients with left ventricular assist devices. J Card Fail 2014;20:899-904.

15. Mabvuure NT, Rodrigues JN. External cardiac compression during cardiopulmonary resuscitation of patients with left ventricular assist devices. Interact Cardiovasc Thorac Surg 2014;19:286-9.

16. Yuzefpolskaya M, Uriel N, Flannery M, et al. Advanced cardiovascular life support algorithm for the management of the hospitalized unresponsive patient on continous flow left ventricular assist device support outside the intensive care unit. Eur Heart J Acute Cardiovasc Care 2016;5: 522-6.

17. Peberdy MA, Gluck JA, Ornato JP, et al. Cardiopulmonary resuscitation in adults and children with mechanical circulatory support: a scientific statement from the American Heart Association. Circulation 2017;135: e1115-34.

18. Raasch H, Jensen BC, Chang PP, et al. Epidemiology, management, and outcomes of sustained ventricular arrhythmias after continuous-flow left ventricular assist device implantation. Am Heart J 2012;164:373-8.

19. Fried J, Garan AR, Shames S, et al. Aortic root thrombosis in patients supported with continuous-flow left ventricular assist devices. J Heart Lung Transplant 2018;37:1425-32.

20. Tanna MS, Reyentovich A, Balsam LB, et al. Aortic root thrombus complicated by left main coronary artery 
occlusion visualized by $3 \mathrm{D}$ echocardiography in a patient with continuous-flow left ventricular assist device. Echocardiography 2017;34:306-10.

21. Spina R, Siriwardena M, Bart N, et al. Primary percutaneous coronary intervention for inferior ST-segment elevation myocardial infarction in a patient supported by the HeartWare left ventricular assist device. Intern Med J 2017;47:1068-71.

22. Dang NC, Topkara VK, Mercando M, et al. Right heart failure after left ventricular assist device implantation in patients with chronic congestive heart failure. J Heart Lung Transplant 2006;25:1-6.

23. Burke MA, Givertz MM. Assessment and management of heart failure after left ventricular assist device implantation. Circulation 2014;129:1161-6.

24. ATLS Subcommittee, American College of Surgeons' Committee on Trauma, International ATLS Working Group. Advanced Trauma Life Support (ATLS $\AA$ ): the ninth edition. J Trauma Acute Care Surg 2013;74:1363-6.

25. Hussmann B, Lendemans S. Pre-hospital and early in-hospital management of severe injuries: changes and trends. Injury 2014;45(Suppl 3):S39-42.

26. Stulak JM, Schettle S, Haglund N, et al. Percutaneous driveline fracture following implantation of the HeartMate II left ventricular assist device: how durable is driveline repair? ASAIO J 2017;63:542-5.

27. Crow S, John R, Boyle A, et al. Gastrointestinal bleeding rates in recipients of nonpulsatile and pulsatile left ventricular assist devices. J Thorac Cardiovasc Surg 2009;137:208-15.

28. Wever-Pinzon O, Selzman CH, Drakos SG, et al. Pulsatility and the risk of nonsurgical bleeding in patients supported with the continuous-flow left ventricular assist device HeartMate II. Circ Heart Fail 2013;6:517-26.

29. Nascimbene A, Neelamegham S, Frazier OH, Moake JL, Dong JF. Acquired von Willebrand syndrome associated with left ventricular assist device. Blood 2016;127:3133-41.

30. Uriel N, Pak SW, Jorde UP, et al. Acquired von Willebrand syndrome after continuous-flow mechanical device support contributes to a high prevalence of bleeding during long-term support and at the time of transplantation. J Am Coll Cardiol 2010;56:1207-13.

31. Crow S, Chen D, Milano C, et al. Acquired von Willebrand syndrome in continuous-flow ventricular assist device recipients. Ann Thorac Surg 2010; 90:1263-9; discussion 1269

32. Sparrow CT, Nassif ME, Raymer DS, Novak E, LaRue SJ, Schilling JD. Pre-operative right ventricular dysfunction is associated with gastrointestinal bleeding in patients supported with continuous-flow left ventricular assist devices. JACC Heart Fail 2015;3:956-64.

33. Draper KV, Huang RJ, Gerson LB. GI bleeding in patients with continuous-flow left ventricular assist devices: a systematic review and meta-analysis. Gastrointest Endosc 2014;80:435-46.e1.

34. Mehra MR, Goldstein DJ, Uriel N, et al. Two-year outcomes with a magnetically levitated cardiac pump in heart failure. N Engl J Med 2018;378:1386-95.

35. Axelrad JE, Pinsino A, Trinh PN, et al. Limited usefulness of endoscopic evaluation in patients with continuous-flow left ventricular assist devices and gastrointestinal bleeding. J Heart Lung Transplant 2018;37:723-32.

36. Aggarwal A, Pant R, Kumar S, et al. Incidence and management of gastrointestinal bleeding with continuous flow assist devices. Ann Thorac Surg 2012;93:1534-40.

37. Demirozu ZT, Radovancevic R, Hochman LF, et al. Arteriovenous malformation and gastrointestinal bleeding in patients with the HeartMate II left ventricular assist device. J Heart Lung Transplant 2011;30:849-53.

38. Hayes HM, Dembo LG, Larbalestier R, O'Driscoll G. Management options to treat gastrointestinal bleeding in patients supported on rotary left ventricular assist devices: a single-center experience. Artif Organs 2010;34:703-6.

39. Draper K, Kale P, Martin B, Cordero K, Ha R, Banerjee D. Thalidomide for treatment of gastrointestinal angiodysplasia in patients with left ventricular assist devices: case series and treatment protocol. J Heart Lung Transplant 2015;34:132-4.

40. Ray R, Kale PP, Ha R, Banerjee D. Treatment of left ventricular assist device-associated arteriovenous malformations with thalidomide. ASAIO J 2014;60:482-3.

41. Acharya D, Loyaga-Rendon R, Morgan CJ, et al. INTERMACS analysis of stroke during support with continuousflow left ventricular assist devices: risk factors and outcomes. JACC Heart Fail 201 7;5:703-11.

42. Parikh NS, Cool J, Karas MG, Boehme AK, Kamel H. Stroke risk and mortality in patients with ventricular assist devices. Stroke 2016;47:2702-6.

43. Harvey L, Holley C, Roy SS, et al. Stroke after left ventricular assist device implantation: outcomes in the continuous-flow era. Ann Thorac Surg 2015;100:535-41.

44. Frontera JA, Starling R, Cho SM, et al. Risk factors, mortality, and timing of ischemic and hemorrhagic stroke with left ventricular assist devices. J Heart Lung Transplant 2017;36:673-83.

45. Godoy DA, Piñero GR, Koller P, Masotti L, Di Napoli M. Steps to consider in the approach and management of critically ill patient with spontaneous intracerebral hemorrhage. World J Crit Care Med 2015;4:213-29.

46. Krstic A, O'Carroll C, Lanza L, Steidley DE, Staley L, Cartin-Ceba R. 1276: simultaneous LVAD thrombosis and ischemic stroke treated successfully with alteplase. Crit Care Med 2015;43:321.

47. Hannan MM, Husain S, Mattner F, et al. Working formulation for the standardization of definitions of infections in patients using ventricular assist devices. J Heart Lung Transplant 2011;30:375-84. 
48. Monkowski DH, Axelrod P, Fekete T, Hollander T, Furukawa S, Samuel R. Infections associated with ventricular assist devices: epidemiology and effect on prognosis after transplantation. Transpl Infect Dis 2007;9:114-20.

49. Miller LW, Pagani FD, Russell SD, et al. Use of a continuous-flow device in patients awaiting heart transplantation. N Engl J Med 2007;357:885-96.

50. Pagani FD, Miller LW, Russell SD, et al. Extended mechanical circulatory support with a continuous-flow rotary left ventricular assist device. J Am Coll Cardiol 2009;54:312-21.

51. Sharma V, Deo SV, Stulak JM, et al. Driveline infections in left ventricular assist devices: implications for destination therapy. Ann Thorac Surg 2012;94:1381-6.

52. Nienaber JJC, Kusne S, Riaz T, et al. Clinical manifestations and management of left ventricular assist device-associated infections. Clin Infect Dis 2013;57:1438-48.

53. Baddour LM, Wilson WR, Bayer AS, et al. Infective endocarditis: diagnosis, antimicrobial therapy, and management of complications: a statement for healthcare professionals from the Committee on Rheumatic Fever, Endocarditis, and Kawasaki Disease, Council on Cardiovascular Disease in the Young, and the Councils on Clinical Cardiology, Stroke, and Cardiovascular Surgery and Anesthesia, American Heart Association: endorsed by the Infectious Diseases Society of America. Circulation 2005;111:e394-434.

54. Sandoe JA, Barlow G, Chambers JB, et al. Guidelines for the diagnosis, prevention and management of implantable cardiac electronic device infection. Report of a joint Working Party project on behalf of the British Society for Antimicrobial Chemotherapy (BSAC, host organization), British Heart Rhythm Society (BHRS), British Cardiovascular Society (BCS), British Heart Valve Society (BHVS) and British Society for Echocardiography (BSE). J Antimicrob Chemother 2015;70:325-59.

55. Mehra MR, Stewart GC, Uber PA. The vexing problem of thrombosis in long-term mechanical circulatory support. J Heart Lung Transplant 2014;33:1-11.
56. Tchantchaleishvili V, Sagebin F, Ross RE, Hallinan W, Schwarz KQ, Massey HT. Evaluation and treatment of pump thrombosis and hemolysis. Ann Cardiothorac Surg 2014;3:490-5.

57. Goldstein DJ, John R, Salerno C, et al. Algorithm for the diagnosis and management of suspected pump thrombus. J Heart Lung Transplant 2013;32:667-70.

58. Sylvia LM, Ordway L, Pham DT, DeNofrio D, Kiernan M. Bivalirudin for treatment of LVAD thrombosis: a case series. ASAIO J 2014;60:744-7.

59. Schrage B, Grahn H, Wagner FM, et al. Effective treatment with a new protocol using tissue-type plasminogen activator thrombolysis for pump thrombosis with the HVAD device. Eur Heart J Acute Cardiovasc Care 2018;7:766-70.

60. Basken R, Bazzell CM, Smith R, Janardhanan R, Khalpey Z. Advantages and disadvantages of using intravenous tissue plasminogen activator as salvage therapy for inoperable HeartWare thrombosis. J Card Surg 201 7;32:443-6.

61. Upshaw JN, Kiernan MS, Morine KJ, Kapur NK, DeNofrio D. Incidence, management, and outcome of suspected continuous-flow left ventricular assist device thrombosis. ASAIO J 2016;62:33-9.

62. Kirklin JK, Naftel DC, Pagani FD, et al. Seventh INTERMACS annual report: 15,000 patients and counting. J Heart Lung Transplant 2015;34:1495-504.

63. Wever-Pinzon O, Givens RC, Flannery M, Naka Y, Jorde UP. Repetitive HeartMate II pump stoppage induced by transitioning from battery to main power source: the short-to-shield phenomenon. J Heart Lung Transplant 2015;34:270-1.

64. Kornberger A, Stock UA, Risteski P, Beiras Fernandez A. Left ventricular non-compaction cardiomyopathy and left ventricular assist device: a word of caution. J Cardiothorac Surg 2016;11:108.

65. Abraham J, Remick JD, Caulfield T, et al. Left ventricular assist device outflow cannula obstruction treated with percutaneous endovascular stenting. Circ Heart Fail 2015;8:229-30. 